\title{
Ink-jet Printing of $\mathrm{YBa}_{2} \mathrm{Cu}_{3} \mathrm{O}_{7}$ Superconducting Coatings and Patterns from Aqueous Solutions
}

Isabel Van Driessche ${ }^{1}$, Jonas Feys ${ }^{1}$, Pieter Vermeir ${ }^{1}$, Petra Lommens ${ }^{1}$

${ }^{1}$ SCRiPTS, Department of Inorganic and Physical Chemistry, Ghent University, Krijgslaan 281 S3, 9000 Gent, Belgium, Isabel.vandriessche@ugent.be.

\begin{abstract}
In this paper, we combine the use of Drop-on-Demand (DOD) ink-jet printing with completely water- based inks as a novel approach to the CSD process for coated conductors. This method holds the promise of improved scalability due to lower ink losses, continuous processing and a drastically increased precursor lifetime due to the prevention of solvent evaporation and dust incorporation. Moreover, ink-jet printing has the potential to switch quite easily from continuous coatings to a multi-filamentary pattern, which is particularly important for alternating current (AC) or field applications of coated conductors. The fluid properties, often expressed with dimensionless constants, like the Reynolds and Weber numbers, for printable liquids were determined. For proof-of-concept, single crystals of $\mathrm{SrTiO}_{3}$ with a low mismatch towards YBCO, were used as substrates.
\end{abstract}

\section{INTRODUCTION}

The production of low cost, long length $\mathrm{YBa}_{2} \mathrm{Cu}_{3} \mathrm{O}_{7-\delta}(\mathrm{YBCO})$ coated conductors is one of the main prerequisites for spreading the use of superconductivity in power applications. Currently, a promising coated conductor design is based on a metallic Ni-5\% W tape (RABiTS) coated with a $\mathrm{La}_{2} \mathrm{Zr}_{2} \mathrm{O}_{7}-\mathrm{CeO}_{2}$ buffer structure, and a superconducting YBCO top layer [1]. Up to now, vacuum techniques have resulted in the best properties for high temperature superconductor (HTSC) thin films. However, to reduce production costs and improve scalability, a shift towards chemical solution deposition (CSD) conditions would be preferred. The main advantages are the lower investment, faster deposition with higher yield, ease of stoichiometric composition control and modification, and processing under ambient pressure, enabling completely continuous production [2].

Inkjet printing is widely used for the fabrication of coatings and patterns onto a variety of substrates . It is a simple and cost-effective technique for ceramic coatings. The reproducible dispensing of ink droplets in the range of $\mathrm{pL}$ to $\mathrm{nL}$ volumes at high rates $(\mathrm{kHz})$ allows for high 3D resolution, strict control of the thickness and gradient porosity. Inkjet systems can be readily scaled-up for industrial manufacturing and the technology is environmentally friendly utilizing only the exact amount of necessary material.

In the present manuscript, drop-on-demand ink-jet printing of coatings and tracks will be reported using piezoelectric printing systems, on both single crystals and industrially-relevant metal substrates, using inks formulated based on non-fluorine chemistries. 


\section{EXPERIMENT}

Water-based precursor solutions were prepared by dissolving stable, cost-effective and easily available inorganic salts in an aqueous solution of coordinating ligands. As a result, solvation by water molecules is discouraged and neither extensive hydrolysis nor precipitation is likely to take place, resulting in very homogeneous materials [3]. The aqueous precursor was prepared starting from $\mathrm{Y}_{2}\left(\mathrm{CO}_{3}\right)_{3} .1 .9 \mathrm{H}_{2} \mathrm{O}\left(99.9 \%\right.$, Sigma Aldrich), $\mathrm{Ba}(\mathrm{OH})_{2} .8 \mathrm{H}_{2} \mathrm{O}(98 \%$, Janssen) and $\mathrm{Cu}\left(\mathrm{NO}_{3}\right)_{2} \cdot 2.5 \mathrm{H}_{2} \mathrm{O}(98 \%$, Alfa Aesar) salts dissolved in water and nitrilo-triacetic acid (NTA, $99 \%$, Alfa Aesar) in a $0.45: 1$ ratio for NTA : total metal concentration. The addition of triethanolamine (99+\%, Acros Organics) increases the $\mathrm{pH}$ and the viscosity to the desired values of 6-8 and $4.77 \mathrm{mPa} \mathrm{s}\left(25^{\circ} \mathrm{C}, 100 \mathrm{rpm}\right.$, Brookfield DV-E Viscometer) respectively. Attention was given towards the development of an ink with neutral $\mathrm{pH}$ and nonaggressive components to prevent corrosion effects inside the printing system. The total metal concentration of the precursor solution was $1.1 \mathrm{~mol} / \mathrm{L}(0.185 \mathrm{~mol} / \mathrm{L} \mathrm{YBCO})$, as verified by ICPOES (Spectro, Genesis). By slow evaporation of the solvent (water) at $60^{\circ} \mathrm{C}$, condensation of the complexes in the solution takes place, leading to the formation of a homogeneous gel network. A stable shelf-life of several months was established. It has been reported previously that this precursor system can be used for several ceramic coatings [4-8], buffer layers [9-10] and superconductors [11]. The samples were heat treated at $20{ }^{\circ} \mathrm{C} / \mathrm{min}$ to $780{ }^{\circ} \mathrm{C}$ for $2 \mathrm{~h}$ in a humid $\mathrm{O}_{2} / \mathrm{N}_{2}$ atmosphere, followed by an oxygenation step at $520^{\circ} \mathrm{C}$, with a dwell at $400{ }^{\circ} \mathrm{C}$.

Laboratory-scale printing was performed using piezoelectric micro-dispensers from Microfab Technologies, Inc. (USA) mounted on X-Y positioning stages under computer control in a clean room environment. Printing was performed by moving the nozzle over the substrate at constant velocity and jetting at a constant frequency (typically $1 \mathrm{kHz}$ ), the ratio of these controlling the inter-droplet spacing (typically $75 \mu \mathrm{m}$ ).

To characterize jetting behaviour and allow the optimisation of ink and printing parameters, an optical drop visualisation system was developed comprising a high-sensitivity camera with $1292 \times 964$ px resolution at 30 frames/s (Allied Vision Technologies, Stingray F125B) and a telecentric zoom lens (ML-Z07545, Moritex). Collimated, strobed LED illumination was used in a backlit configuration. The camera shutter and LED strobe were synchronized with droplet ejection with a selectable delay time, such that each frame corresponded to the state of the ink stream a chosen time after ejection.

The critical temperature of the superconducting layers was measured by resistivity measurements as a function of temperature using a custom-made four-point test device (Keithley). The critical current was determined from the third harmonic of the induced signal in a pick-up coil from an AC drive signal, using a Theva Cryoscan setup in liquid nitrogen. For this inductive measurement, a constant-voltage criterion of $50 \mu \mathrm{V}$ was selected.

The composition, crystallinity and texture of the processed films was verified using X-ray diffraction, both in the Bragg-Brentano configuration for phase identification and configured for texture analysis (Thermo Scientific ARL X'TRA and Siemens D5000; $\mathrm{Cu}-\mathrm{K}_{\alpha}$ ). The sample morphology was characterized using optical microscopy (Leitz, Laborlux 12 POL S) and SEM (FEI Quanta 200 FEG). A cross section of the layers was made using a FIB module coupled with 
SEM to verify the thickness of the layers. The topology of multi-filamentary patterns was visualized using optical and AFM profilometry (Veeco NT9080).

\section{DISCUSSION}

The generation of droplets in a DOD printer is a complex process, and the precise physics and fluid mechanics of the process are the subject of much research [12]. The behaviour of inks in the printing system can be quantified by a number of dimensionless groupings of physical constants, i.e. the Reynolds ( $\mathrm{Re})$, Weber (We) and Ohnesorge (Oh) numbers:

$R e=\frac{v r \rho}{\eta}, W e=\frac{v^{2} r \rho}{\sigma}, O h=\frac{\sqrt{W e}}{R e}=\frac{\eta}{\sqrt{\sigma \rho r}}$

with $\sigma, \rho, \eta$ and $v$ the ink surface tension $\left(\mathrm{J} \mathrm{m}^{-2}\right)$, density $\left(\mathrm{kg} \mathrm{m}^{-3}\right)$, viscosity (Pa s) and velocity $\left(\mathrm{m} \mathrm{s}^{-1}\right)$ respectively and $r$ the radius of the orifice of the nozzle $(\mathrm{m})$. The Reynolds number is a ratio of internal and viscous forces and the Weber number shows the ratio between internal and surface tension forces. The inverse value of the Ohnesorge number is a characteristic dimensionless number which is independent of droplet velocity. Often it is written that $\mathrm{Oh}^{-1}$ should be between 1 and 10 for proper jetting properties. If the ratio is too low, viscous forces become more dominant preventing drop ejection; conversely, if the ratio is too high the possibility for satellite droplet formation becomes high [13-15]. In Table 1, we show the different numbers calculated for our ink, taking into account the ink viscosity obtained at the highest shear rate. For $\mathrm{Oh}^{-1}$, a value of 7.37 is calculated, which is within the desired range.

Table I. Fluid properties of the YBCO ink for an orifice radius (r) of $3 \times 10^{-5} \mathrm{~m}$.

\begin{tabular}{lllllllll}
\hline \multirow{2}{*}{ Type of ink } & $\begin{array}{l}\text { Surface } \\
\text { tension } \\
\sigma\left[\mathrm{J} \mathrm{m}^{-2}\right]\end{array}$ & $\begin{array}{l}\text { Density } \\
\rho\left[\mathrm{kg} \mathrm{m}^{-}\right.\end{array}$ & $\begin{array}{l}\text { Viscosity } \\
\eta[\mathrm{Pa} \mathrm{s}]\end{array}$ & $\begin{array}{l}\text { Orifice } \\
\text { diameter } \\
(\mu \mathrm{m})\end{array}$ & Re & We & $O^{-1}$ \\
\hline Water-based & $\begin{array}{l}6.79 \times \\
10^{-2}\end{array}$ & 1233 & $6.8 \times 10^{-3}$ & 30 & 15.77 & 4.58 & 7.37
\end{tabular}

From Figure 1, one can see that initially, the drops form a liquid column which transforms into the actual droplet and an elongated tail. Here, breaking up of this tail from the droplet leads to the formation of a satellite drop. The presence of these satellite droplets should be avoided at impact with the substrate, as the key goal is to leave a single isolated droplet to optimize precision, resolution and accuracy during printing. Therefore, the distance between the nozzle and the substrate should be chosen in such a way that the two droplets can merge before impact. On the other hand, an increased standoff will reduce the accuracy, because drag from air currents in the printing chamber makes the droplets deviate from their vertical trajectory, so the distance should be set as low as possible. In our case, the optimal distance between print head and substrate was determined to be between $0.6 \mathrm{~mm}$ and $2 \mathrm{~mm}$. 


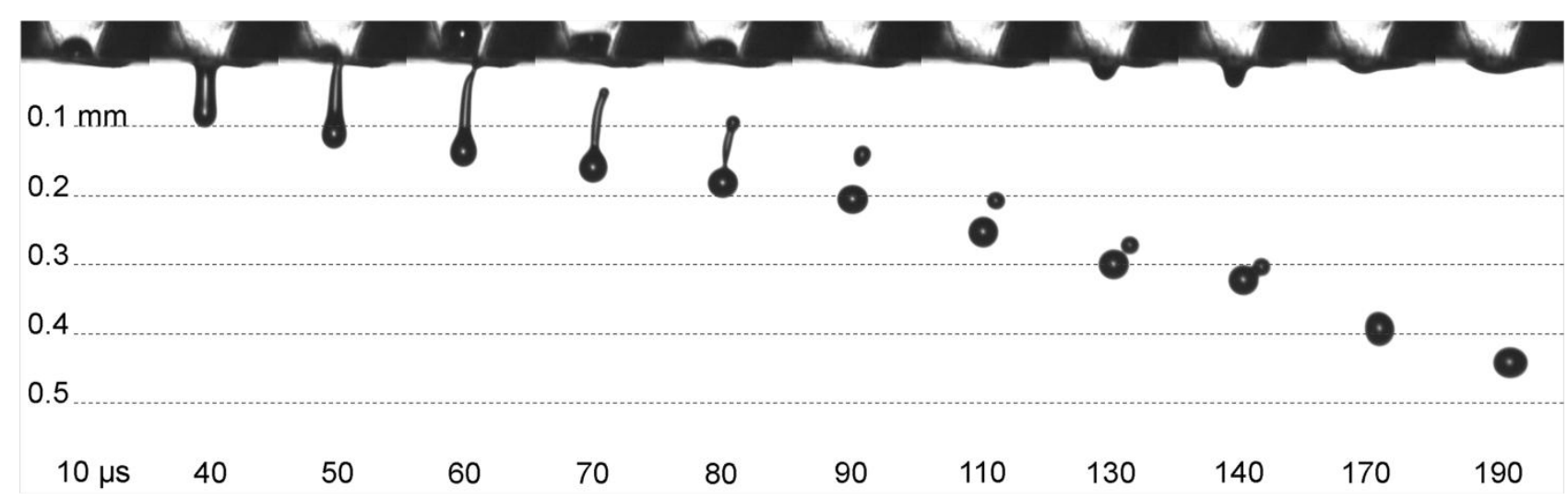

Figure 1. Jetting analysis by strobe imaging as a function of time after ejection of the YBCO ink from the nozzle.

The estimated volume and velocity of droplets are within the range of 65 to $80 \mathrm{pl}$ and 2.3 to 3.5 $\mathrm{m} / \mathrm{s}$ respectively within the printing parameters tested. For the experiment displayed in Figure 1, the volume is around $75 \mathrm{pl}$ with a droplet velocity of $2.9 \mathrm{~m} / \mathrm{s}$ after merging at $190 \mu \mathrm{s}$. At $110 \mu \mathrm{s}$, the estimated volumes for the main droplet and the satellite are close to $60 \mathrm{pl}$ and $15 \mathrm{pl}$ respectively.

In figure 2, optical and profilometry images are presented of printed wet droplets, dried at $100^{\circ} \mathrm{C}$. The diameters and heights of the droplets in the two left images vary in the ranges 145 $160 \mu \mathrm{m}$ and $40-160 \mathrm{~nm}$ respectively and, for the pattern, between $120-140 \mu \mathrm{m}$ and $170-200$ $\mathrm{nm}$ respectively. Due to the small volume of material in one droplet or a single printed line, with rapid solvent evaporation from the edges, a clear "coffee-ring" effect can be observed in the profilometry measurements.

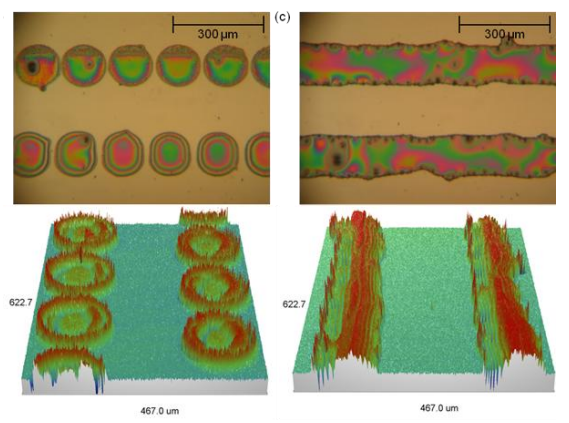

Figure 2. Optical micrographs and interference profilometry profiles for droplets of the YBCO water-based ink, printed with longitudinal spacings of (a) $0.2 \mathrm{~mm}$, (b) $0.15 \mathrm{~mm}$ and (c) $0.1 \mathrm{~mm}$, demonstrating that both continuous coating and patterning are possible.

When bringing the droplets closer together in the axial direction, it becomes equally possible to change from printing a pattern to achieving complete coverage of the substrate. The thickness after complete heat treatment of the YBCO thin films varies between 310 and $400 \mathrm{~nm}$.

Microstructural investigation of the top layer by SEM, is showing a crack-free and dense surface. Though, secondary phases enriched by $\mathrm{Ba}$ and $\mathrm{Cu}$, are visible. The morphology will be further improved by an optimisation of the heat treatment. 
XRD patterns reveal the formation of crystalline films of pure YBCO (figure 3). The in-plane and out-of-plane misorientation of the YBCO film was further characterized by a $\varphi$-scan (figure $3 \mathrm{~b}$ ) and $\omega$-scan (figure $3 \mathrm{a}$, inset). An average full width at half maximum (FWHM) value of $1.87^{\circ}$ for the (103) $\varphi$-scan and a FWHM of $0.68^{\circ}$ for the (005) $\omega$-scan proves that highly textured YBCO was obtained.
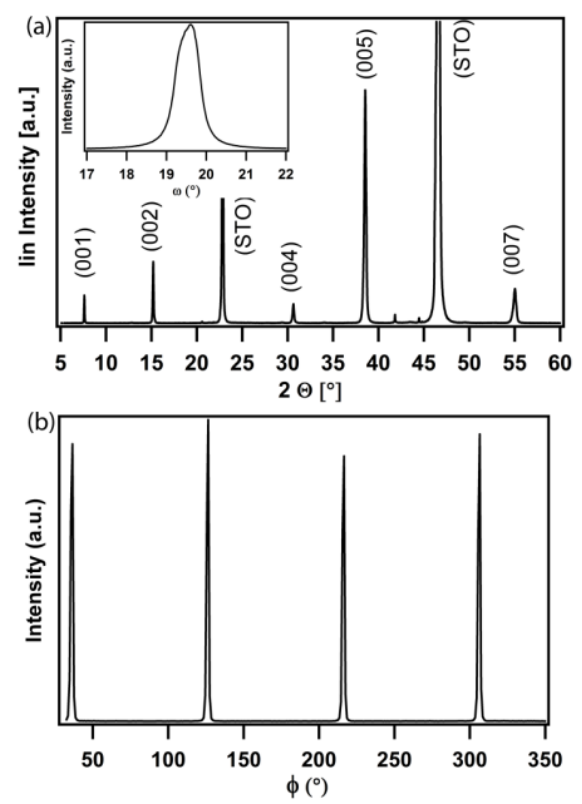

Figure 3. (a) $\theta-2 \theta$ scan and $\omega$-scan of the (005) plane (inset) and (b) $\varphi$-scan obtained for the (103) plane of the water-based YBCO ink after complete conversion with an average FWHM for the last two of $0.68^{\circ}$ and $1.87^{\circ}$ respectively.

The result evidences a high critical temperature $T_{\mathfrak{c}(50)} \geq 92 \mathrm{~K}$ and a sharp transition into the superconducting state. A Jc of $0.7 \mathrm{MA} / \mathrm{cm}^{2}$ was determined.

\section{CONCLUSIONS}

We have successfully deposited complete coatings and multi-filamentary structures of YBCO on a single crystal $\mathrm{SrTiO}_{3}$ substrate using a DOD piezoelectric ink-jet printing system. A new and stable YBCO ink was prepared starting from low cost and environmentally benign metal salts. This ink was characterized for its jetting properties as well as for the wetting on the substrate. With a ratio $\mathrm{Re} / \mathrm{We}^{1 / 2}$ of 7.37 and a surface tension of the liquid within the five degree wetting envelope for a cleaned $\mathrm{SrTiO}_{3}$ substrate, the ink rheology is well within the criteria for good jetting and wetting behaviour. After optimization, printing of multi-filamentary YBCO patterns became possible by carefully tuning the printing parameters. Tracks $200 \mathrm{~nm}$ thick and $200 \mu \mathrm{m}$ wide, were deposited and heat treated. The tracks have sharp edges and are very homogeneous in their overall profile, showing almost no coffee ring effect. A full YBCO coating with a final thickness of $310-400 \mathrm{~nm}$ and exhibiting strong c-axis orientation was also obtained, with a critical current density of $0.67 \mathrm{MA} / \mathrm{cm}^{2}\left(\mathrm{I}_{\mathrm{c}}=23.5 \mathrm{~A} / \mathrm{cm}\right)$ at $77 \mathrm{~K}$ in self-field. 


\section{ACKNOWLEDGMENTS}

This research was funded by the European Union under the FP7 Frame work : EU project EFECTS (FP7-NMP-2007-SMALL-1 grant n²05854).

\section{REFERENCES}

1. K. Knoth, S. Engel, C. Apetrii, M. Falter, B. Schlobach, R. Hühne, S. Oswald, L. Schultz, B. Holzapfel, Current Opinion in Solid State and Materials Science 2006, 10, 205.

2. Van Driessche I, Penneman G, Bruneel E, Hoste S, Pure Appl Chem, 2002, 74, 2101-9.

3. Van Driessche I, Penneman G, Abell JS, Bruneel E, Hoste S, Thermec'2003, Pts 1-5, 2003, 426-432, 3517-22.

4. De Buysser K, Lommens P, de Meyer C, Bruneel E, Hoste S, Van Driessche I, 2004, 48, 139-44.

5. Gryglewicz G, Stolarski M, Gryglewicz S, Klijanienko A, Piechocki W, Hoste S, Van Driessche I, Carleer R, Yperman J, 2006, 62, 135-41.

6. Vergote GJ, Vervaet C, Van Driessche I, Hoste S, De Smedt S, Demeester J, Jain RA, Ruddy S, Remon JP, Int J Pharm, 2002, 240, 79-84.

7. Le MT, Van Well WJM, Van Driessche I, Hoste S, Applied Catal A, 2004, 267, 227-34.

8. M. Arin, P. Lommens, N. Avci, S. C. Hopkins, K. De Buysser, I. M. Arabatzis, I. Fasaki, D. Poelman and I. Van Driessche, J. Eur. Ceram. Soc. 31, 1067-1074 (2011).

9. Penneman G, Van Driessche I, Bruneel E, Hoste S, Euro Ceramics Viii, Pts 1-3, 2004, 264-268, 501-4.

10. Van Driessche I, Penneman G, De Meyer C, Stambolova I, Bruneel E, Hoste S, Ttp, Euro Ceramics Viii, Pts 1-3, 2002, 206-2, 479-82.

11. J. Feys, P. Vermeir, P. Lommens, S. C. Hopkins, X. Granados, B. A. Glowacki, M. Baecker, E. Reich, S. Ricard, B. Holzapfel, P. Van der Voort and I. Van Driessche, J. Mater. Chem. 22, 3717-3726 (2012).

12. B. Derby, in Annual Review Of Materials Research, Vol. 40, Annual Reviews, Palo Alto 2010, 395.

13. B. Derby, N. Reis, K. A. M. Seerden, P. S. Grant, J. R. G. Evans, Solid Freeform and Additive Fabrication-2000 2000, 625, 195.

14. R. Noguera, M. Lejeune, T. Chartier, J Eur Ceram Soc 2005, 25, 2055

15. J. Windle, B. Derby, J Mater Sci Lett 1999, 18, 87. 